\title{
Phase Transitions of Bosons in Optical Lattices with a Mixture of Single and Pair Hoppings
}

\author{
V.M. TRAvin And T.K. KOpEĆ* \\ Institute of Low Temperature and Structure Research, Polish Academy of Sciences, \\ P.O.B. 1410, 50-950 Wrocław, Poland
}

\begin{abstract}
We considered Bose condensate in optical lattice with mixture of single and pair hoppings for arbitrary temperatures. In order to calculate free energy of the system and determine phase transition lines between disordered and ordered phases, the Laplace transform method has been applied. We identified several possible scenarios for phase diagrams with phase transitions of the second kind. The results have been obtained from both analytical and numerical methods of calculation. Finally, we obtained thermal insensitivity of the system for big values of reduced pair hopping and (or) reduced chemical potential.
\end{abstract}

DOI: 10.12693/APhysPolA.130.625

PACS/topics: $67.10 . \mathrm{Fj}, 67.85 . \mathrm{Hj}, 67.90 .+\mathrm{z}$

\section{Introduction}

Trapping of bosons in optical lattices is possible due to AC Stark effect. Interaction between bosons and electromagnetic field strongly depends from the state of the atoms and polarisation of the electromagnetic field [1]. Atom in the state ${ }^{2} S_{1}$ possesses 2 possible quantum numbers $m_{J}= \pm 1 / 2$. If one radiates left-handed polarized light $\sigma^{-}$on the ${ }^{2} S_{1}$ atom with $m_{J}=1 / 2$ he obtaines atom with excited states ${ }^{2} P_{3 / 2}$ and ${ }^{2} P_{1 / 2}$ for which the Stark effect has the same power but different signs; as a result, total Stark effect is 0. On the other hand, righthanded polarized light $\sigma^{+}$creates only one excited state ${ }^{2} P_{3 / 2}$ and total Stark effect is non-zero [2]. It means that atoms in ground state with $m_{J}= \pm 1 / 2$ have non-zero total Stark effect only for $\sigma^{ \pm}$polarizations. This feature leads to existence of so-called state-dependent lattices that have two orthogonal states $(+)$ and $(-)$ containing interacting bosons. It is possible to achieve a monochromatic radiation where all photons have the same polarization, that is so-called well defined polarization, and create an optical superlattice with two sublattices with right- and left-handed polarized light [3]. Once particles get into the superlattice they separate between two sublattices due to theirs $m_{J}= \pm 1 / 2$ states. Two particles constantly interacting with each other within one site can change their state to the opposite one. That means once two particles collide they do not more experience interaction with their current sublattice anymore. As a result, two particles move to the nearest site with another state. This is a mechanism of the pairing hopping proposed in [4]. We solved $p$-particle hopping problem where $p$ is an arbitrary integer value in [5] and this work is an expansion where we combined both mechanisms of particles movement in optical lattices and studied their behaviour at arbitrary temperatures.

\footnotetext{
${ }^{*}$ corresponding author; e-mail: t.kopec@int.pan.wroc.pl
}

\section{Model}

The second-quantized bosonic Hubbard Hamiltonian of the ultracold atoms in a state-dependent optical lattice with $N$ number of sites reads [6]:

$$
\begin{aligned}
\hat{\mathcal{H}} & =\frac{U}{2} \sum_{i} \hat{n}_{i}\left(\hat{n}_{i}-1\right)-J_{1} \sum_{\langle i, j\rangle}\left(\hat{a}_{i}^{\dagger} \hat{a}_{j}+\text { H.c. }\right) \\
& -J_{2} \sum_{\langle i, j\rangle}\left(\hat{a}_{i}^{\dagger} \hat{a}_{i}^{\dagger} \hat{a}_{j} \hat{a}_{j}+\text { H.c. }\right)-\mu \sum_{i} \hat{n}_{i},
\end{aligned}
$$

where index $i=1,2, \ldots, N$ stands for number of the site, summation $\langle i, j\rangle$ is done over the nearest neighbors with a coordination number $z$, first term represents twobody interaction, second term - basic single hopping, third term is pair hopping and the last term describes interaction with environment. Operators $\hat{a}_{i}^{\dagger}$ and $\hat{a}_{i}$ are operators of creation and annihilation of the particle in the site $i$, respectively. Operator $\hat{n}_{i}$ is a density operator $\hat{n}_{i}=\hat{a}_{i}^{\dagger} \hat{a}_{i}$ of the particles in site $i$. $U$ is a two-body interaction energy, $J_{1}$ is a single hopping energy, $J_{2}$ is a pair hopping energy and $\mu$ is a chemical potential that controls number of particles in the system.

We used mean-field theory of the creation and annihilation operators considering them as fluctuations near their expectation values.

$$
\begin{array}{ll}
\hat{a}_{i}^{\dagger}=\Phi_{i}+\delta_{i}^{\dagger}, & \hat{a}_{i}^{\dagger} \hat{a}_{i}^{\dagger}=\Psi_{i}+\eta_{i}^{\dagger}, \\
\hat{a}_{i}=\Phi_{i}+\delta_{i}, & \hat{a}_{i} \hat{a}_{i}=\Psi_{i}+\eta_{i},
\end{array}
$$

where $\Phi_{i}$ and $\Psi_{i}$ are order parameters for single and pair hoppings, respectively, and can be calculated as statistical averages

$$
\Phi_{i}=\left\langle\hat{a}_{i}\right\rangle, \quad \Psi_{i}=\left\langle\hat{a}_{i} \hat{a}_{i}\right\rangle,
$$

where we use a definition of the statistical average as follows:

$$
\langle\ldots\rangle=\frac{\operatorname{Tr}\left(\ldots \mathrm{e}^{-\beta \hat{H}}\right)}{\operatorname{Tr} \mathrm{e}^{-\beta \hat{H}}},
$$

where $\beta=\frac{1}{k_{B} T}, k_{B}$ is the Boltzmann constant and $T$ is a temperature. 
After a substitution of the mean-field operators representation into the model Hamiltonian one obtaines meanfield Hamiltonian (1) as follows:

$$
\begin{aligned}
& \hat{\mathcal{H}}=N \hat{H}, \quad \hat{H}=\hat{\mathcal{U}}+\hat{V}, \\
& \hat{\mathcal{U}}=\frac{U}{2} \hat{n}(\hat{n}-1)-\mu \hat{n}-J_{2} z \Psi\left(\hat{a} \hat{a}+\hat{a}^{\dagger} \hat{a}^{\dagger}\right), \\
& \hat{V}=-J_{1} z \Phi\left(\hat{a}+\hat{a}^{\dagger}\right),
\end{aligned}
$$

where $\hat{H}$ is a one site mean-field Hamiltonian and $N$ is a number of sites. Value $z$ is a coordination number.

In this case one can calculate partition function of the system

$$
\mathcal{Z}=\left(\operatorname{Tr} \mathrm{e}^{-\beta \hat{H}}\right)^{N} .
$$

When one knows partition function it is easy to calculate free energy

$$
f=-\frac{1}{\beta N} \ln \mathcal{Z} \text {. }
$$

The feature of this problem is that one has two order parameters $\Phi$ and $\Psi$ for single and pair hoppings, respectively, having only one mixture of bosons. Partition function $\mathcal{Z}$ includes only diagonal terms of the matrix exponent. It means that terms like $\left(J_{1}\right)^{2} J_{2} z^{3} \Phi^{2} \Psi \hat{a} \times a \times \hat{a}^{\dagger} \hat{a}^{\dagger}$ also have nonzero contribution. Finally, free energy is nonsymmetric upon $\Phi \leftrightarrow \Psi$ transformation. Based on the said above we can write down first terms of free energy without direct calculation

$$
\begin{aligned}
f & =f_{0}+A_{1} \Phi^{2}+A_{2} \Psi^{2}+B \Phi^{2} \Psi \\
& +C_{1} \Phi^{4}+C_{2} \Psi^{4}+D \Phi^{2} \Psi^{2}+\ldots
\end{aligned}
$$

In order to build phase transition lines one has to find minima of a free energy. It can be done via self-consistent equations calculation

$$
\begin{aligned}
\frac{\partial f}{\partial \Phi} & =\Phi\left\{\left[J_{1} z-\frac{1}{\beta} \frac{Z_{2}^{\Phi}}{Z_{0}}\right]-\frac{1}{\beta} \frac{Z_{3}}{Z_{0}} \Psi\right. \\
- & \left.\frac{2}{\beta}\left[\frac{Z_{4}^{\Phi}}{Z_{0}}-\frac{\left(Z_{2}^{\Phi}\right)^{2}}{2 Z_{0}^{2}}\right] \Phi^{2}-\frac{1}{\beta} \frac{Z_{4}^{\Phi \Psi}}{Z_{0}} \Psi^{2}\right\}=0, \\
\frac{\partial f}{\partial \Psi} & =-\frac{1}{\beta} \frac{Z_{3}}{Z_{0}} \Phi^{2}+\Psi\left\{\left[J_{2} z-\frac{1}{\beta} \frac{Z_{2}^{\Psi}}{Z_{0}}\right]\right. \\
-\frac{2}{\beta} & {\left.\left[\frac{Z_{4}^{\Psi}}{Z_{0}}-\frac{\left(Z_{2}^{\Psi}\right)^{2}}{2 Z_{0}^{2}}\right] \Psi^{2}-\frac{1}{\beta} \frac{Z_{4}^{\Phi}}{Z_{0}} \Phi^{2}\right\}=0 . }
\end{aligned}
$$

Self-consistent Eq. (10) breaks symmetry of solutions between $\Phi$ and $\Psi$, because it cannot be satisfied for a situation when $(\Phi \neq 0, \Psi=0)$. In this case, there are 3 out of 4 possible solutions for order parameters

$$
\begin{aligned}
& \Phi=0, \quad \Psi=0 ; \\
& \Phi=0, \quad \Psi \neq 0 ; \\
& \Phi \neq 0, \quad \Psi \neq 0 .
\end{aligned}
$$

In this case we have to calculate phase transition line for the second region, because first region is given by isolated single and pair hoppings problems considered to be known and the third region can be obtained by exceptional principle.

The idea of calculations lies in the Laplace transform method. Partition function can be written down in the following way:

$$
\begin{aligned}
& \mathcal{Z}=\mathrm{e}^{-N \beta J_{1} z \Phi^{2}-N \beta J_{2} \Psi^{2}}\left[\mathcal{Z}^{\prime}\right]^{N}, \\
& \mathcal{Z}^{\prime}=\int_{\Gamma} \frac{\mathrm{d} s}{2 \pi \mathrm{i}} \mathrm{e}^{\beta s} \operatorname{Tr}\left[\frac{1}{s+\hat{\mathcal{U}}+\hat{V}}\right] .
\end{aligned}
$$

It can be shown that expression under the integral can be transformed as follows [7, 8]:

$$
\begin{aligned}
\mathcal{Z}^{\prime}-\mathcal{Z}_{0}=\beta \int_{0}^{1} \frac{\mathrm{d} g}{g} \int_{\Gamma} \frac{\mathrm{d} s}{2 \pi \mathrm{i}} \\
\times \mathrm{e}^{\beta s} \operatorname{Tr}\left[\sum_{k=1}^{\infty}(-1)^{k}\left[g(s+\hat{\mathcal{U}})^{-1} \hat{V}\right]^{k}\right],
\end{aligned}
$$

where $\mathcal{Z}_{0}=\operatorname{Tr} \mathrm{e}^{-\beta \hat{\mathcal{U}}}$.

The last representation of the partition function can be read as direct expansion of the partition function with respect to order parameter $\Phi$ that is small near the phase transition line of the $\Phi=0, \Psi \neq 0$ region while $\Psi$ is arbitrary value.

We expanded free energy up to forth degree of the order parameter $\Phi$ and numerically calculated coefficients of the expansion

$$
\begin{aligned}
f & =-\frac{1}{\beta} \ln Z_{0}(\Psi)+\left[J_{1} z-\frac{1}{\beta} \frac{Z_{2}(\Psi)}{Z_{0}(\Psi)}\right] \Phi^{2} \\
& +\frac{1}{\beta}\left[\frac{Z_{4}(\Psi)}{Z_{0}(\Psi)}-\frac{Z_{2}^{2}(\Psi)}{2 Z_{0}^{2}(\Psi)}\right] \Phi^{4},
\end{aligned}
$$

where $Z_{2}(\Psi)$ can be obtained from the second order of the partition function expansion $(13)$, and $Z_{4}(\Psi)$ from the fourth order of the same partition function expansion.

The idea behind the numerical calculations is a truncated Hamiltonian that can be represented as a matrix and substituted into the partition function expansion (13). Order parameter $\Psi$ was calculated also numerically from its definition.

In this model free energy depends on 5 physical values: $T, J_{1}, J_{2}, U, \mu$. First, we fix two-body interaction and relates other values to the energy $U$. It means that we consider dimensionless parameters that are so-called reduced single hopping $z J_{1} / U$ etc. Second, we study phase diagrams at fixed temperature. Finally, we fix reduced pair hopping and look on phase diagrams for the single hopping. It has been done due to a fact that phase transition line is given by equation that can be obtained from the coefficient before $\Phi^{2}$ in (14):

$$
\frac{J_{1} z}{U}=\beta \frac{\mathcal{Z}_{0}(\mu)}{\mathcal{Z}_{2}\left(1, J_{2}, \mu\right)},
$$

where $\mathcal{Z}_{2}\left(J_{1}, J_{2}, \mu\right)$ is a quadratic terms coefficient of partition function expansion. This equation is correct due to the fact that $\mathcal{Z}_{2}\left(J_{1}, J_{2}, \mu\right) \propto J_{1}^{2} z^{2}$.

We should notice that this equation also satisfies for pair hopping phase transition line. The last formula is 
transcended with respect to pair hopping energy $J_{2}$ and it makes sense to produce phase diagrams for single hopping with arbitrary values of $J_{2}$ and then invert function $J_{1}=$ $J_{1}\left(J_{2}\right) \rightarrow J_{2}=J_{2}\left(J_{1}\right)$.

\section{Results}

We calculated phase transition lines of the bosonic system at different temperatures. Analysis shows us that we have 3 different phase transition lines of the second kind: (i) between $\Phi=0, \Psi=0$ and $\Phi=0, \Psi \neq 0$; (ii) between $\Phi=0, \Psi=0$ and $\Phi \neq 0, \Psi \neq 0$; (iii) between $\Phi=0, \Psi \neq 0$ and $\Phi \neq 0, \Psi \neq 0$.

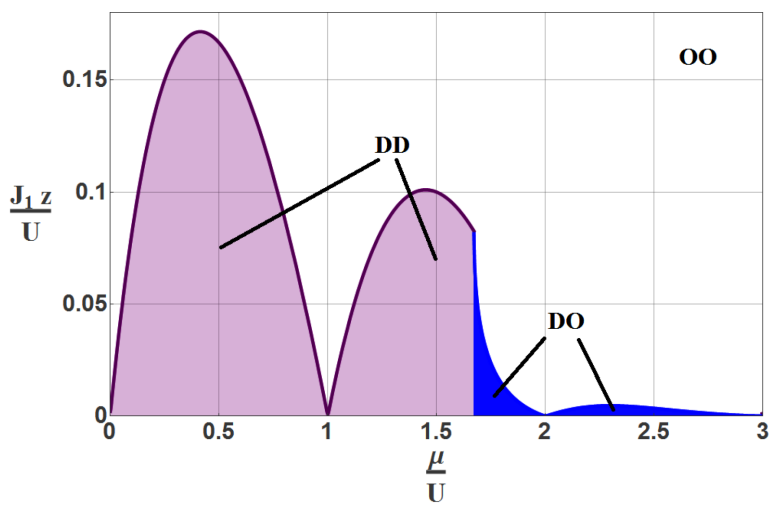

Fig. 1. Phase transition line for the single hopping with a fixed pairing hopping $J_{2} / U=0.075$ at zero temperature. DD region stands for disordered-disordered phase $\Phi=0, \Psi=0$, DO region stands for disorderedordered phase $\Phi=0, \Psi \neq 0$ and OO stands for orderedordered phase $\Phi \neq 0, \Psi \neq 0$. On the edge between DD and $\mathrm{OO}$ phases one has a straight phase transition line of a second kind for the pair hopping.
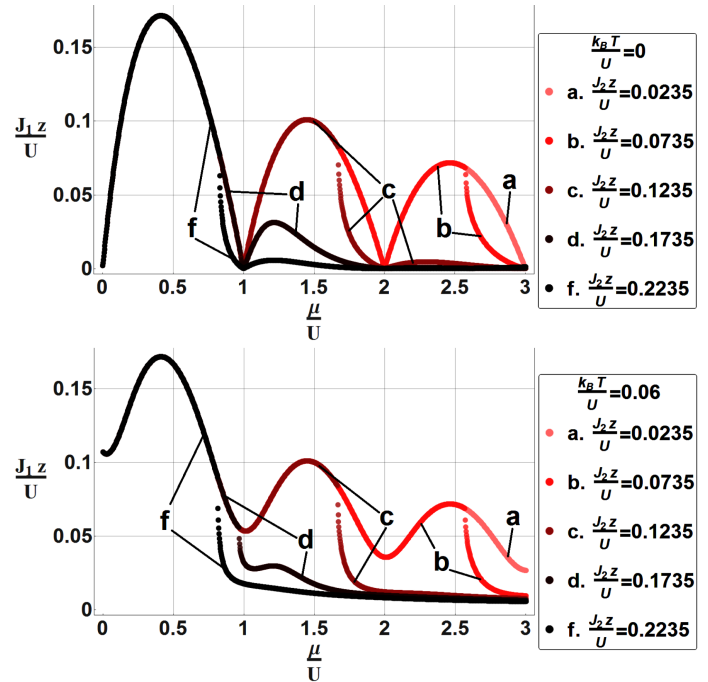

Fig. 2. Set of the phase transition lines at zero temperature (above) and at $k_{B} T / U=0.06$ (below) with different values of the pair hopping energies. For $k_{B} T / U=$ 0.06 phase transition lines with different values of $J_{2} / U$ go to the close value with increase of the chemical potential. Increase of the pair hopping leads to the shift of the edge between DD and DO phases to the smaller values of reduced chemical potential.

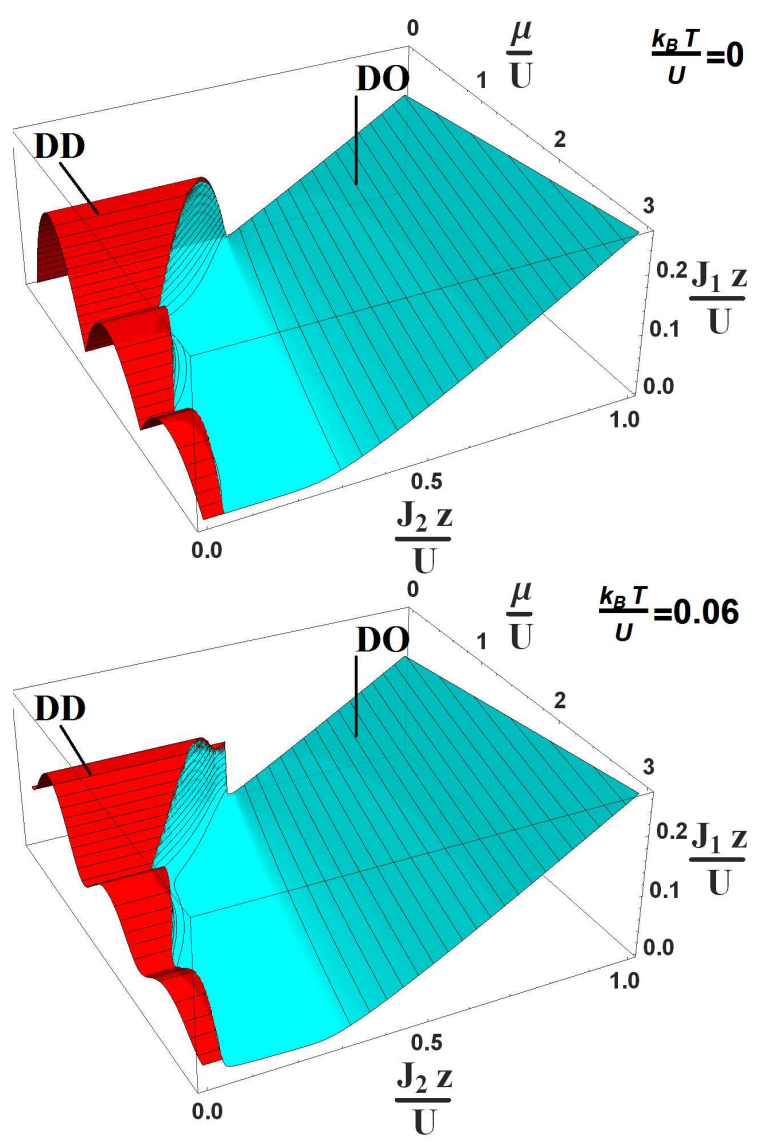

Fig. 3. Phase transition surface created by combination of the set of the phase transition lines with fixed pair hopping. As DD we represent phase transition surface between disordered-disordered phase (below the surface) and ordered-ordered phase (above the surface); as DO we represent phase transition surface between disordered-ordered phase (below the surface) and ordered-ordered phase (above the surface). Phase transition surface between disordered-disordered and disordered-ordered phases matches the surface that can be created upon projection of the boundary between DD and DO onto the $\left(J_{2} z / U, \mu / U\right)$ plane.

As one can see in Fig. 1 for DD region phase transition line matches pure single hopping problem while appearance of the pairing hopping in DO leads to decrease of the disordered phase region near the critical point. Nevertheless, with increase of the chemical potential pairing hopping leads to increase of the disordered phase region in general.

As mentioned before we fixed temperature and twobody interaction. After the fixation free energy can be represented as a function of 3 variables which means that one has not a phase transition line but phase transition surface. In Fig. 2 one can see cross-sections of that surface. In our work we built huge amount of cross-sections and their combination lets us reproduce full picture that one can see in Fig. 3. Finally, due to the independence and equality of single and pair hoppings we reproduce 


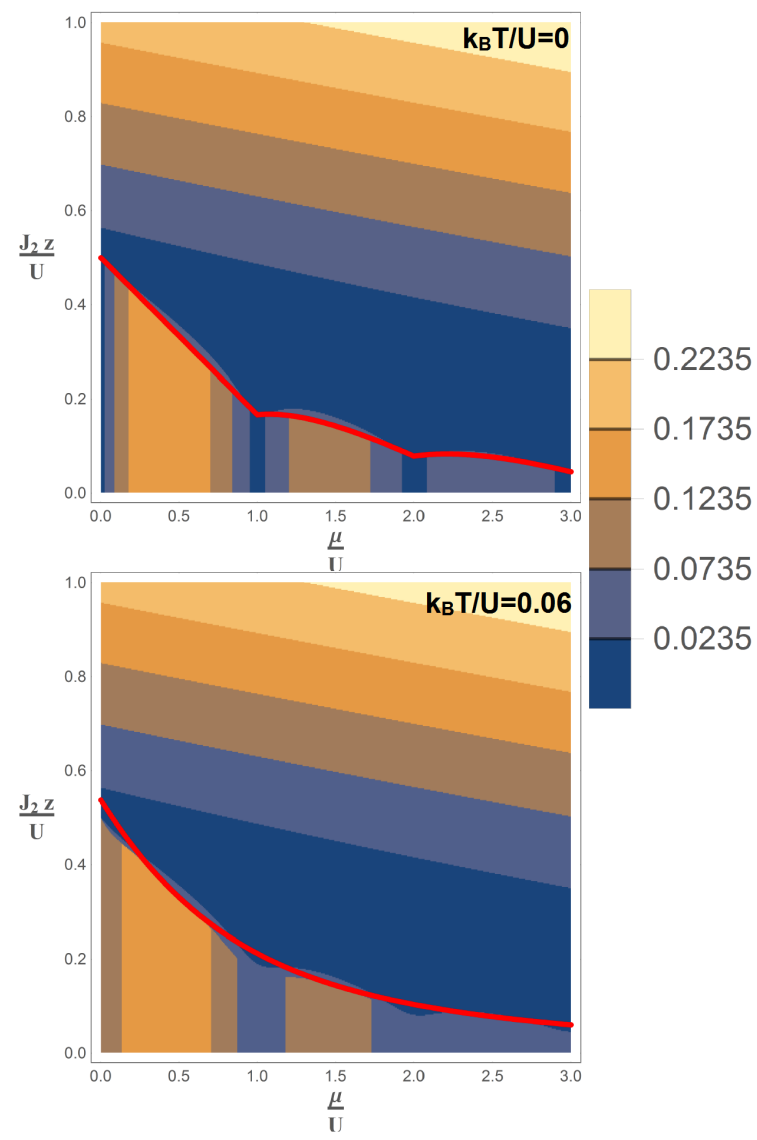

Fig. 4. Phase transition lines of the pair hopping with a fixed single hopping. In our representation colour regions stand for single hopping disordered phase and their edges are phase transition lines of the second kind for single hopping. The line PH PT stands for pair hopping phase transition line in such way that below this line one has disordered phase for the pair hopping and ordered phase above. Total phase of the system can be obtained by overlay of the disordered and ordered regions for both hoppings. One should remember that OD cannot exist. On the legends one has values of the single hopping energies $J_{1} z / U$. a dependence of a reduce pair hopping $J_{2} z / U$ from a reduced chemical potential $\mu / U$ on the Fig. 4 that in fact are cross-sections of the phase transition surface.

\section{Conclusions}

We studied behaviour of the bosonic atoms in a homogeneous state-dependent optical lattice. It was possible to build phase transition surface in $J_{1} z / U, J_{2} z / U$ and $\mu / U$ axes and study behaviour of the phase transition with a temperature increase. Upon increase of the chemical potential for the fixed pair hopping we obtained vanishing of the loops and spread of the disordered single hopping phase. Next, we found out a thermal independence of the system for big values of reduced pair hopping or(and) reduced chemical potential. We suppose that one should consider bigger temperatures in order to observe changing of the phase transition surface in discussed region, but one still can say that pair hopping leads to the thermal stability of the system and it becomes less sensitive to the temperature changing.

\section{References}

[1] I.H. Deutsch, P.S. Jessen, Phys. Rev. A 57, 1972 (1998).

[2] D. Jaksch, H.-J. Briegel, J.I. Cirac, C.W. Gardiner, P. Zoller, Phys. Rev. Lett. 82, 1975 (1999).

[3] S. Trotzky, P. Cheinet, S. Fölling, M. Feld, U. Schnorrberger, A.M. Rey, A. Polkovnikov, E.A. Demler, M.D. Lukin, I. Bloch, Science 319, 295 (2008).

[4] M. Eckholt, J.J. Garcia-Ripoll, New J. Phys. 11, 093028 (2009).

[5] V.M. Travin, T.K. Kopeć, J. Phys. A Math. Theor. 48, 345001 (2015).

[6] Xiang-Fa Zhou, Yong-Sheng Zhang, Guang-Can Guo, Phys. Rev. A 80, 013605 (2009).

[7] H. Keiter, G. Morandi, Phys. Rep. 5, 227 (1984).

[8] T.K. Kopeć, M.W. Szymański, Phys. Lett. A 378, 3402 (2014). 